\title{
Measurements of Water-Air Flow Phenomena in a Chamber with a Rotating Shaft
}

\section{TIUTIURSKI Pawel ${ }^{1}{ }^{*}$, HERCEL Paulina ${ }^{1 *}$, TELEGA Janusz $^{2}$, KARDAŚ Dariusz $^{1}$}

1. Flow and Combustion Department, Renewable Energy Faculty, Institute of Fluid Flow Machinery Polish Academy of Sciences, Fiszera 14, 80-231, Gdańsk, Poland

2. Flow and Combustion Department, Experimental Aerodynamics Faculty, Institute of Fluid Flow Machinery Polish Academy of Sciences, Fiszera 14, 80-231, Gdańsk, Poland

(C) The Author(s) 2020

\begin{abstract}
The paper concerns the phenomena of the fluid film that occurs in the bearing chamber of an aircraft engine. The geometry of the system includes two concentric cylinders, between which there is a mixture of oil and air. The fluid circulates in a closed circuit. The rotary movement of the inner cylinder causes creation of the fluid film on the walls of the chamber. The article proposes a measurement method that is allowing observation of this fluid film and, in particular, analysis of the movement of the air bubbles occurring in the film. An own model of the bearing chamber with transparent walls was constructed for the research. For the investigation, water was used instead of oil. Observation of the behaviour of the flow was possible thanks to video recordings made with the use of a fast-capture camera. The results presented in the paper include velocity magnitudes of the air bubbles in the fluid film in dependence on the rotational speed of the shaft and water volume fraction and with a range from 0.37 to $0.91 \mathrm{~m} / \mathrm{s}$. The results presented in this article can be used for the bearing chamber numerical models validation.
\end{abstract}

\section{Keywords: bearing chamber, measurements, fluid film velocity, two-phase flow}

\section{Introduction}

During the work of an engine, heat transfer between the walls and the oil film occurs. To provide safe and reliable operation of the bearing chamber, it is important to investigate and know the behaviour of the air/oil flow, particularly the HTC (heat transfer coefficient) magnitudes. Because HTC is highly dependent on fluid film speed, it is also crucial to know the values of the film velocity.

The purpose of the research was to measure the speed of the air bubbles existing in the fluid film in the bearing chamber. A model of the bearing chamber with a two-phase water-air flow instead of oil-air flow occurring in the original bearing chamber was constructed. Using water for the experiments made the measurement method easier and cleaner than it would be using oil.

Glahn et al. [1] chose three theoretical models and tried to describe the fluid flow in a bearing chamber with these models. One of the models assumed a laminar character of the flow and two other models assumed a turbulent character. For their calculations, they needed to 


\begin{tabular}{lll}
\hline \multicolumn{2}{l}{ Nomenclature } & \\
$\varepsilon$ & BR & bottom right, falling side \\
$\omega \quad$ water volume fraction $/ \%$ & TL & top left, rising side \\
Positions on the bearing chamber & TR & top right, falling side \\
BL $\quad$ bottom left, rising side & & \\
\hline
\end{tabular}

determine the velocity of the fluid flow and used the LDV (Laser Doppler Velocimetry) method. The investigation was carried out for oil flow of $150 \mathrm{~L} / \mathrm{h}$ and for shaft rotational speeds of 3000 and $12000 \mathrm{r} / \mathrm{min}$. The best correlation was obtained for the Deissler turbulent model.

In 1999, Glahn et al. [2] confirmed their previous results [1] with the use of the LDA (Laser Doppler Anemometry) method. Once again, the authors proved that fluid film flow can be described quite accurately with the Deissler turbulent model. It was observed that the increase of the shaft rotational speed causes more uniform distribution of the fluid film and a decrease of the local oil flux at the bottom of the chamber.

Gorse et al. [3] carried out measurements of the height and velocity of the fluid film in a bearing chamber model for different operational conditions. To determine the velocity, the LDV method was used. The results showed strong dependence of the local height and velocity on the bearing chamber geometry (location of the vents) and operational conditions, particularly the flow rate of the air and oil.

Wang et al. [4] proposed a model implemented in MATLAB environment. Their calculations were twodimensional. The model assumed that the fluid film is so thin that its height is negligible regarding the radius of the chamber. The presence and interactions of the oil droplets in the air-phase were taken into consideration. Height and velocity of the film amounted to, respectively, ( 8.5 to 10.5$) \times 10^{-4} \mathrm{~m}$ and $3 \mathrm{~m} / \mathrm{s}$ to $3.7 \mathrm{~m} / \mathrm{s}$ for the shaft rotational speeds of $4000 \mathrm{r} / \mathrm{min}$ to $15000 \mathrm{r} / \mathrm{min}$.

The significant difference between water and oil film results from the difference in viscosity of these fluids. Water has a much lower viscosity than oil, which causes faster turbulence of the water film and changes its flow properties and heat exchange conditions. For this reason, the paper of Deng et al. [5] was devoted to the issue of modeling eddy viscosity in turbulent water films. An extension of this subject is found in the work of Deng et al. [6], where the thickness of the viscous sublayer and the dimensionless wall distance $y^{+}$were determined.

Several studies were made toward better understanding of the two-phase oil/air flow in a bearing chamber over the past three decades. Most of them, however, focus directly on the heat transfer coefficient. Despite of many papers describing measurements and numerical calculations of the parameters of the flow in the bearing chamber, there is not much information about the fluid film velocity. The purpose of this investigation is to analyze the fluid film behaviour by observation of the air bubbles appearing in the film.

\section{Experimental Setup}

The main part of the IMP Raginis stand-up presented in Fig. 1 is the model of the bearing chamber. The model consists of an electric motor with a special shaft installed and transparent walls, so that it was possible to observe the fluid flow. The measurement equipment includes a fast-capture camera (Fastec Imaging HiSpec 2G Mono camera and Nikon AF DC-Nikkor camera lens) and a lighting set-up (halogen lamp and softbox). In the experiments, water is used instead of oil, since it is much cleaner and easier to apply in the experiments. Water is

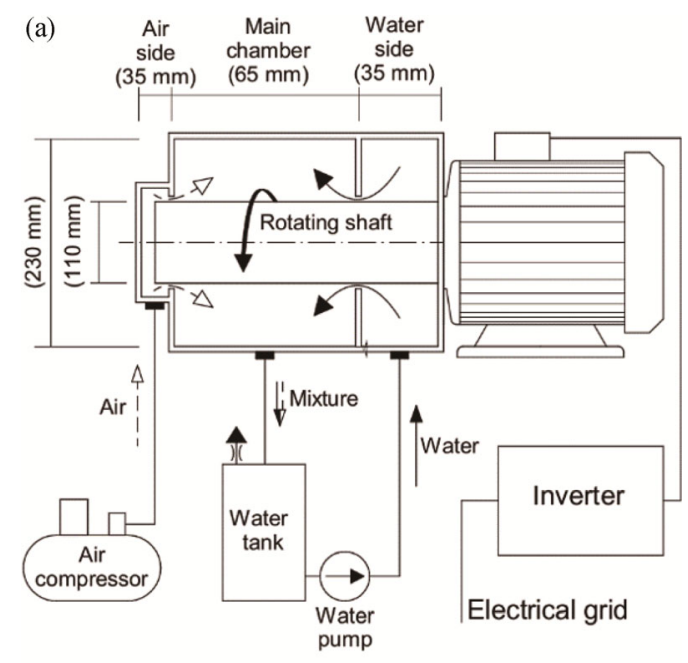

(b)

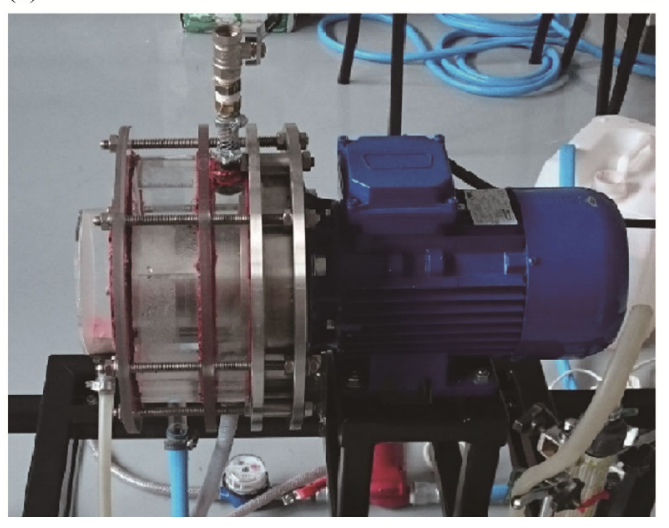

Fig. 1 The experimental set-up 
supplied by a water pump and the air flow is provided by an air compressor. The water-air mixture leaves the model of the bearing chamber by the outlet placed on the bottom of the chamber, owing to the height difference. The shaft rotational speed is controlled by a frequency inverter.

Investigation of the fluid flow was conducted for different water flow rates and shaft rotational speeds. Nine measuring points were determined with measurement conditions presented in Table 1. Water volume fraction is the percent of volume occupied by the water in the water-air mixture flowing into the chamber. The air flow rate was constant for each case and amounted to $2.5 \mathrm{~m}^{3} \cdot \mathrm{h}^{-1}$.

With the use of the fast-capture camera, for each measuring point four video recordings were captured at four different positions on the wall of the Raginis stand-up. The locations of these points are shown in Fig. 2. Each film lasts $3.95 \mathrm{~s}$. The frame rate of the video is 2889 fps. A sample frame is shown in Fig. 3. A linear millimeter scale that can be observed in the figure was

Table 1 Measuring conditions

\begin{tabular}{cccc}
\hline No. & $\begin{array}{c}\text { water volume } \\
\text { fraction } / \%\end{array}$ & $\begin{array}{c}\text { water flow } \\
\text { rate } / \mathrm{m}^{3} \cdot \mathrm{h}^{-1}\end{array}$ & $\begin{array}{c}\text { shaft rotational } \\
\text { speed } / \mathrm{r} \cdot \mathrm{min}^{-1}\end{array}$ \\
\hline 1 & 15.00 & 0.44 & 2000 \\
2 & 15.00 & 0.44 & 1500 \\
3 & 15.00 & 0.44 & 1000 \\
4 & 17.04 & 0.51 & 2000 \\
5 & 17.04 & 0.51 & 1500 \\
6 & 17.04 & 0.51 & 1000 \\
7 & 21.43 & 0.68 & 2000 \\
8 & 21.43 & 0.68 & 1500 \\
9 & 21.43 & 0.68 & 1000 \\
\hline
\end{tabular}

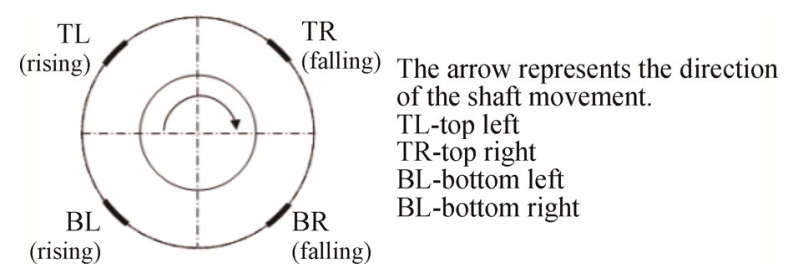

Fig. 2 Measuring conditions

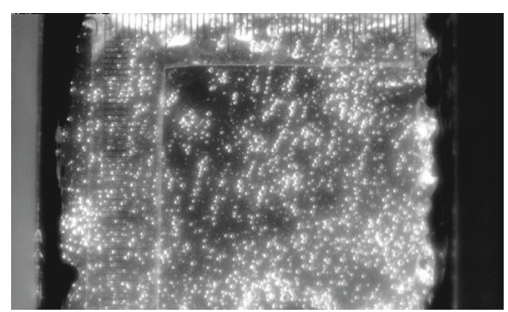

Fig. 3 Sample frame with a linear millimeter scale stuck to the wall stuck to the wall of the model in order to calculate the real displacement of the air bubbles.

Thanks to presence of air, the energy of rotating shaft was transferred to the water and the effect was generation of the fluid film on the walls of the bearing chamber. In the investigated case, regardless of the shaft rotational speed and water volume fraction, air bubbles were observed in the fluid film and were used to investigate the flow.

To analyze the frames obtained from the experiments conducted, software written in the MATLAB environment was used. The program bases its analyses on a comparison of the level of brightness of the pixels for each subsequent frame.

\section{Frame Analysis}

To analyze the frames obtained due to the experiments conducted, a software written in the MATLAB environment was used. The program bases on a comparison of brightness degree of the pixels for each further frame. Scheme presented on Fig. 4 explains the algorithm of the software. On the mentioned figure two frames are presented. Each cell represents one pixel (real frames consist of $560 \times 336$ pixels). Numbers from 1 to 9 symbolize degrees of brightness of each pixel.

The frame analysis procedure is as follows: a set of frames and an air bubble on the first frame are chosen. The software reads the selected bubble as one pixel and defines an area around it (black bold line on Fig. 4.). The dimensions of the area can be given before the programme starts and are dependent of the size of the bubble. The software reads and records degrees of brightness of each pixel in this area. In the next step, the programme analyses every possible position of the air bubble in the second frame in the area around the bubble (area marked with a red line on Fig. 4). The size of the analyzed area is determined by the user before the program starts. The software calculates the correlation between the matrix made from the brightness degrees from the first step with all the possible matrixes found in the second step. The middle pixel of the area that obtained a maximum of correlation is marked as the position of the chosen bubble on the next frame. Every change of the position of the air bubble can be checked and corrected manually if needed, as shown on Fig. 5.

In the experiments, fifteen air bubbles for each video, which yields 60 air bubbles for a measuring case, were analysed. For better accuracy of the results, the air bubbles chosen for analysis were characterized by a long exposure time. The data obtained from the software were averaged to obtain the mean velocity of the bubbles for 


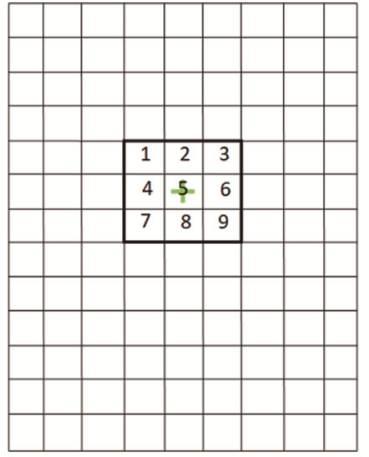

frame 1

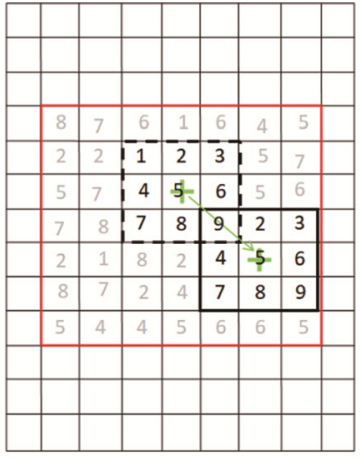

frame 2
Fig. 4 Scheme explaining the algorithm of the software used in the analysis

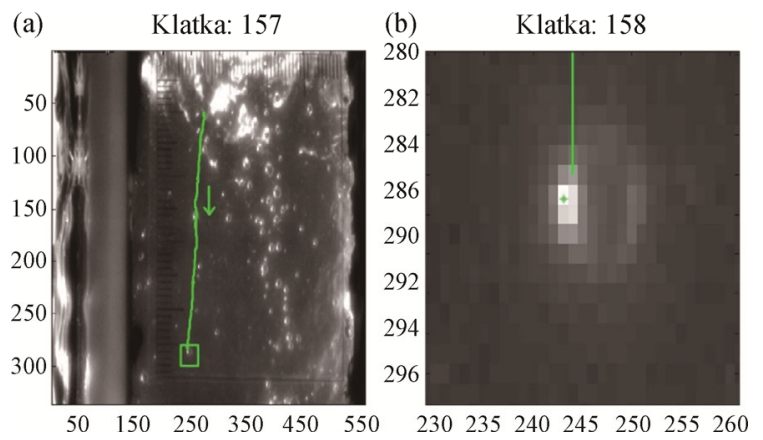

Fig. 5 Manual correction of the air bubble position (entire frame on the left and zoomed area marked with the green rectangle on the right)

each of the films. In other words, for each measuring point, four average velocity magnitudes of the air bubbles for the four positions at the model of the chamber (Fig. 2) were obtained.

\section{Experimental Results}

\subsection{Trajectories of air bubbles}

Figs. 6 and 7 show trajectories of sample air bubbles with a water volume fraction of $15 \%$ and a shaft rotational speed of $2000 \mathrm{r} / \mathrm{min}$. It can be observed that on the right (falling) side (TR, BR - Fig. 2) of the Raginis bearing chamber model, the movement of all air bubbles takes place in the same direction: from the top of the model to the bottom with a small inclination to the air side. On the left (rising) side (TL, BL), however, the motion of the bubbles is more curved and different for each bubble. This relation is observed regardless of the water volume fraction and shaft rotational speed.

As can be seen from Figs. 3, 6, and 7, the mean size of the bubbles was circa $1 \mathrm{~mm}$ in diameter. What is unusual is that it was observed that the movement of the bubbles always took place from the top to the bottom of the chamber, even for the left (rising) side.
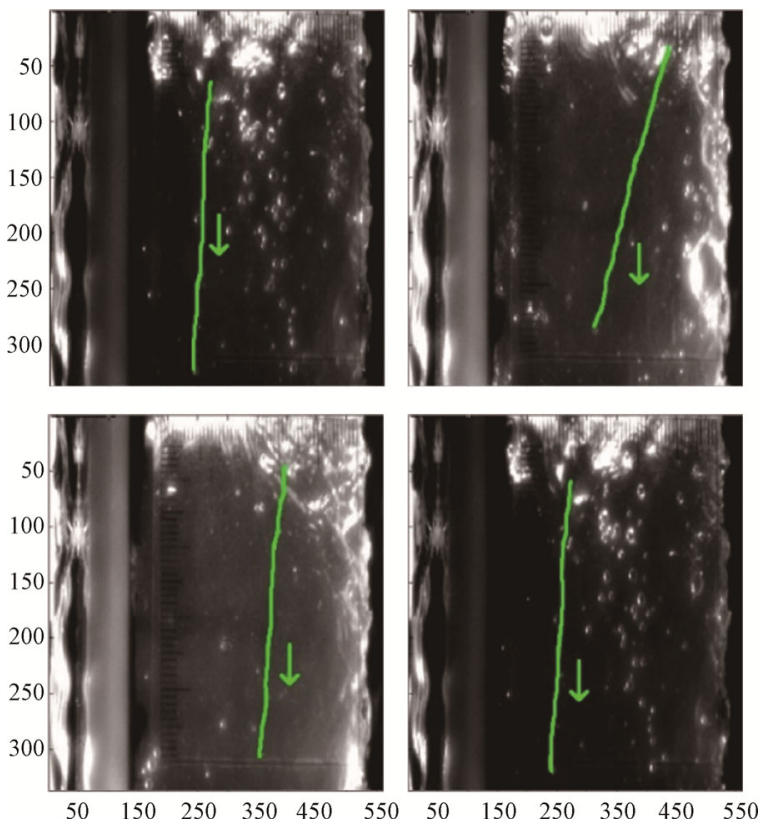

Fig. 6(a) Trajectories of the sample air bubbles on the top right side of the bearing chamber model
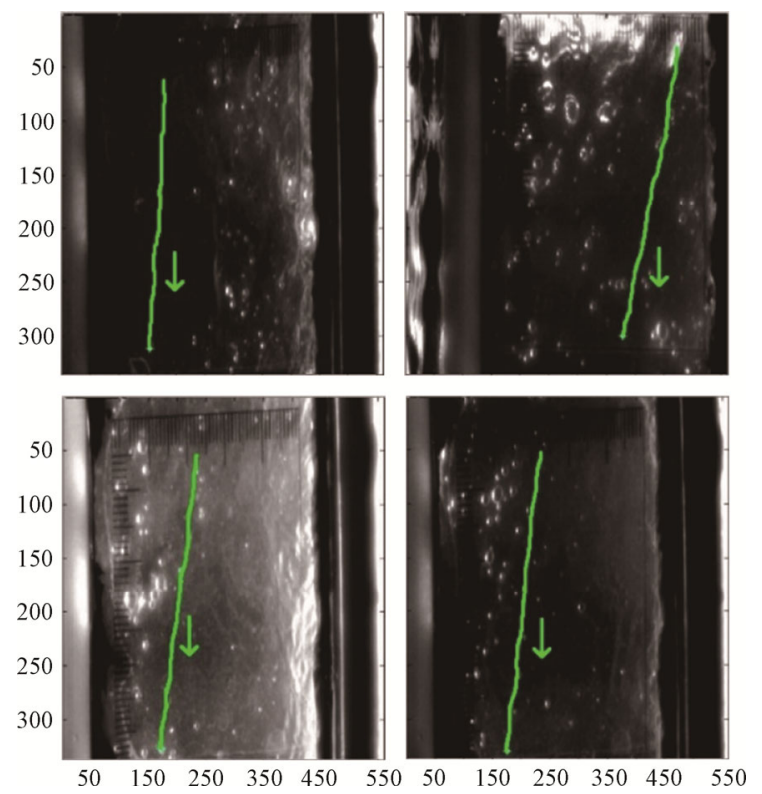

Fig. 6(b) Trajectories of the sample air bubbles on the bottom right side of the bearing chamber model

\subsection{Mean velocities}

Mean velocities of the air bubbles for each of the locations and each of the measuring points are presented in Table 2. Figs. 8 and 9 present graphical display of the results obtained.

It can be observed that an increase of the shaft rotational speed causes smaller differences between the velocities of the air bubbles on the four walls of the chamber. The average velocity magnitudes of the air 
bubbles on the right (falling) side of the chamber (TR, $\mathrm{BR}$ ) are generally higher than on the left (rising) side of the chamber (TL, TR), which is a result of the need to overcome the gravitational force in the beginning of the movement by the bubbles on the left side (in experiments the shaft rotation was clockwise, see Fig. 2). The values of the velocities of the air bubbles are higher at the bottom of the chamber (BL, BR) than on the top. At both sides of the chamber, the trajectory of the bubbles goes from the top to the bottom, so that this relation is a result

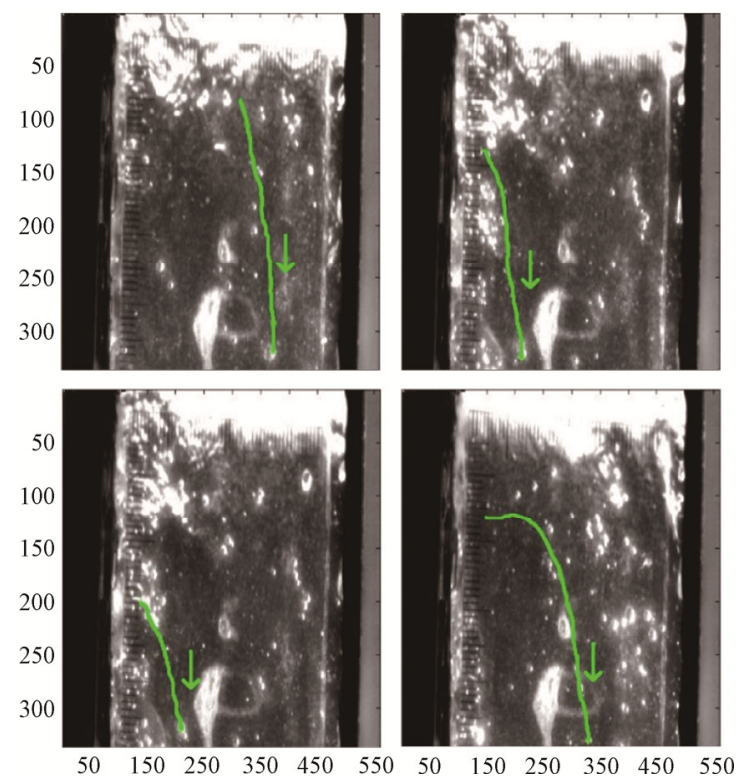

Fig. 7(a) Trajectories of the sample air bubbles on the top left side of the bearing chamber model
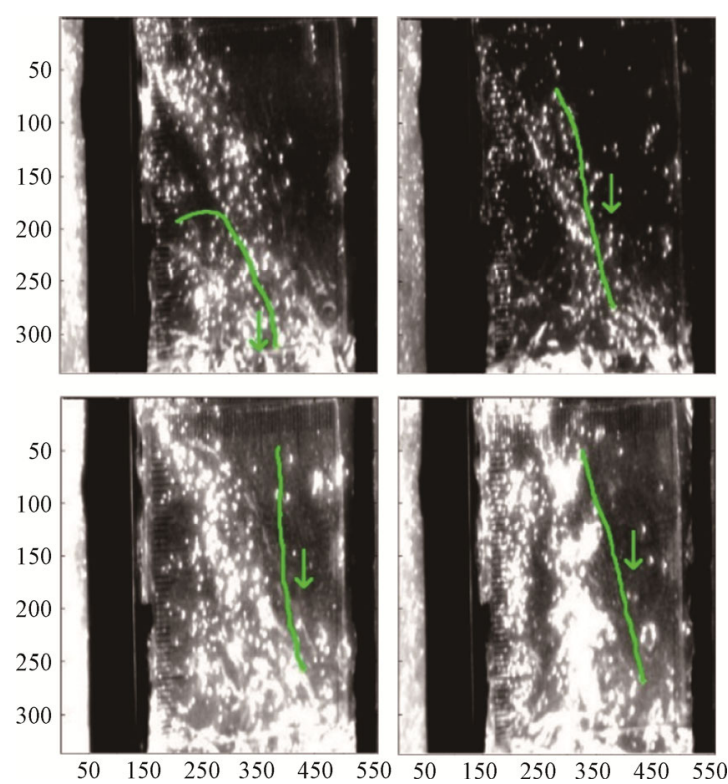

Fig. 7(b) Trajectories of the sample air bubbles on the bottom left side of the bearing chamber model of the gravitational force acting on the air-water mixture and conversion of the potential energy of the bubble at the top into the kinetic energy of the bubble at the bottom.

Table 2 Experimental results

\begin{tabular}{cccccc}
\hline & & & \multicolumn{3}{c}{ average velocity $/ \mathrm{m} \cdot \mathrm{s}^{-1}$} \\
\cline { 5 - 6 }$\varepsilon / \%$ & $\omega / \mathrm{r} \cdot \mathrm{min}^{-1}$ & $\mathrm{TR}$ & $\mathrm{BR}$ & $\mathrm{TL}$ & $\mathrm{BL}$ \\
\hline 15.00 & 2000 & 0.54 & 0.87 & 0.45 & 0.61 \\
15.00 & 1500 & 0.54 & 0.86 & 0.46 & 0.56 \\
15.00 & 1000 & 0.51 & 0.86 & 0.37 & 0.44 \\
17.04 & 2000 & 0.56 & 0.69 & 0.45 & 0.51 \\
17.04 & 1500 & 0.50 & 0.62 & 0.44 & 0.57 \\
17.04 & 1000 & 0.74 & 0.63 & 0.42 & 0.52 \\
21.43 & 2000 & 0.70 & 0.81 & 0.46 & 0.50 \\
21.43 & 1500 & 0.80 & 0.64 & 0.45 & 0.48 \\
21.43 & 1000 & 0.79 & 0.70 & 0.39 & 0.91 \\
\hline
\end{tabular}

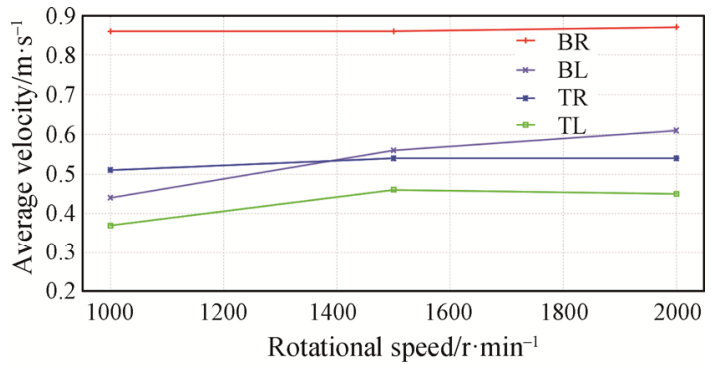

(a) Water volume fraction $15 \%$

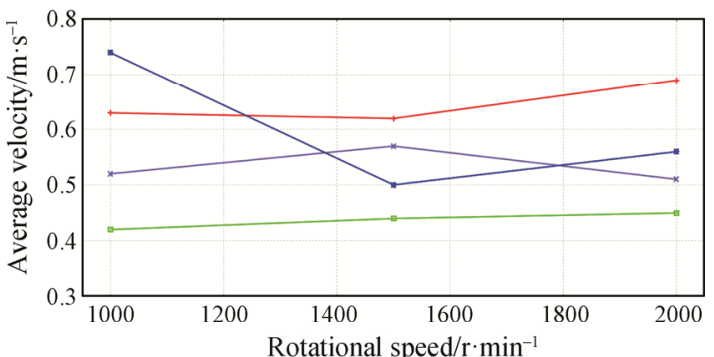

(b) Water volume fraction $17.04 \%$

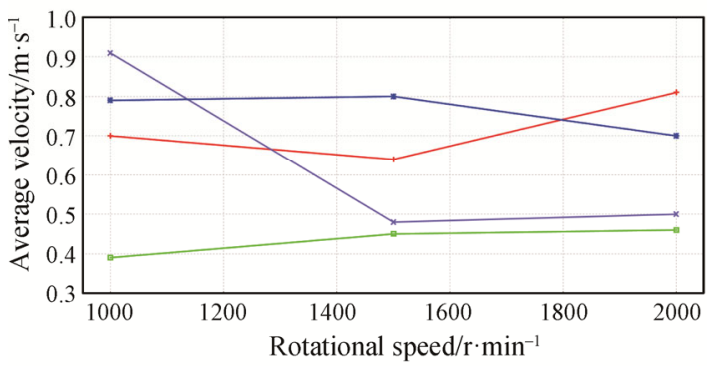

(c) Water volume fraction $21.43 \%$

Fig. 8 Dependence between the average velocity of the air bubbles and the rotational speed at constant water volume fraction 


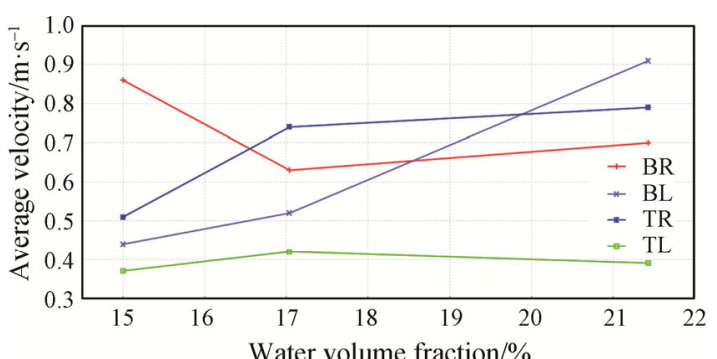

(a) $1000 \mathrm{r} / \mathrm{min}$

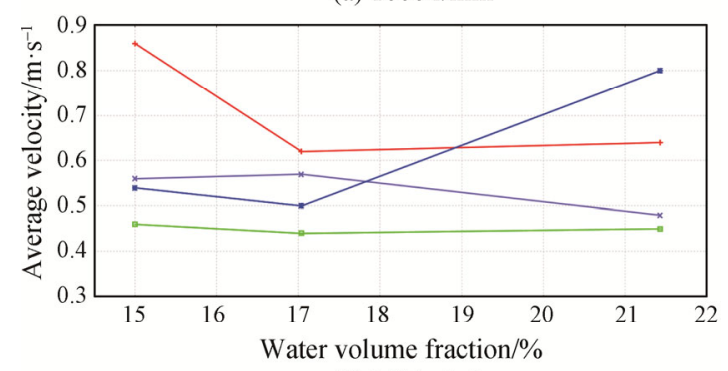

(b) $1500 \mathrm{r} / \mathrm{min}$

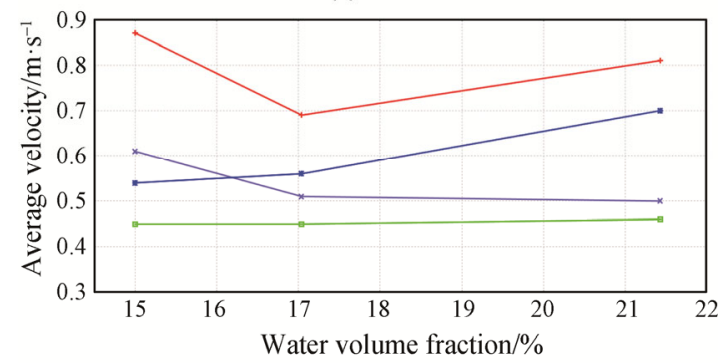

(c) $2000 \mathrm{r} / \mathrm{min}$

Fig. 9 Dependence between the average velocity of the air bubbles and the water volume fraction at constant rational speed

\section{Conclusion}

On the basis of the results and observations, the following conclusions can be drawn. The speed of the air bubbles ranged from 0.37 to $0.91 \mathrm{~m} / \mathrm{s}$. Higher velocity values were observed at the bottom of the bearing chamber than at the top. Similarly, higher velocity magnitudes were observed on the right (falling) side (in accordance with the shaft rotation direction) than on the left (rising) side of the bearing chamber model. On both sides of the bearing chamber, the movement of the bubbles takes place in one direction: from the top to the bottom; however on the rising side (where the motion of the bubbles was opposite to the movement of the shaft) it was more curved and different for each bubble.

To conclude, the research was an attempt to measure the speed of the air bubbles appearing in the film in the bearing chamber. Based on these results, it is possible to estimate the speed of the fluid film in the bearing chamber. The results presented in this article can be used for validation of the numerical model of the bearing chamber. Thereafter, verified numerical models can be used to calculate real-working bearing chambers with oil instead of water. As long as the fluid film velocity is one of the parameters that determine the heat transfer between the fluid and the wall of the bearing chamber, knowing these values will allow calculation of the HTC magnitudes with greater accuracy.

\section{Acknowledgment}

The research for this paper was financially supported by the E-BREAK - Engine Break- through Components and Subsystems under grant agreement No. 314366.

\section{References}

[1] Glahn A., Wittig S., Two-phase air/oil flow in aero engine bearing chambers: Characterization of oil film flows. Journal of Engineering for Gas Turbines and Power, 1996, 118: 578-583.

[2] Glahn A., Wittig S., Two-phase air/oil flow in aero engine bearing chambers - Assessment of an analytical prediction method for the internal wall heat transfer. International Journal of Rotating Machinery, 1999, 5(3): 155-165.

[3] Gorse P., Busam S., Dullenkopf K., Influence of operating condition and geometry on the oil film thickness in aero-engine bearing chamber. Journal of Enginering for Gas Turbines and Power, 2004, 128(1): 103-110.

[4] Wang C., Morvan H.P., Hibberd S., Cliffe K.A., Thin film modelling for aero-engine bearing chambers. Proceedings of the ASME turbo expo 2011, vol 1, ASME Turbo Expo 2011, Vancouver, Canada, June 6-10, 2011.

[5] Deng X., Weaver B., Watson C., Branagan M., Wood H., Fittro R., Modeling Reichardt's formula for eddy viscosity in the fluid film of tilting pad thrust bearings. Journal of Engineering for Gas Turbines and Power-Transactions of the ASME, 2018, 140(8): 082505. Paper No: GTP-17-1614.

[6] Deng X., Gates H., Fittro R., Wood H., Methodology of turbulence parameter correction in water-lubricated thrust bearings. Journal of Fluids Engineering-Transactions of the ASME, 2019, 141(7): 071104. Paper No: FE-18-1452. 